\title{
Structural and spectral properties of minimal strong digraphs
}

\author{
C. Marijuán \\ J. García-López , L.M. Pozo-Coronado
}

\begin{abstract}
In this article, we focus on structural and spectral properties of minimal strong digraphs (MSDs). We carry out a comparative study of properties of MSDs versus trees. This analysis includes two new properties. The first one gives bounds on the coefficients of characteristic polynomials of trees (double directed trees), and conjectures the generalization of these bounds to MSDs. As a particular case, we prove that the independent coefficient of the characteristic polynomial of a tree or an MSD must be $-1,0$ or 1 . For trees, this fact means that a tree has at most one perfect matching; for MSDs, it means that an MSD has at most one covering by disjoint cycles. The property states that every MSD can be decomposed in a rooted spanning tree and a forest of reversed rooted trees, as factors. In our opinion, the analogies described suppose a significative change in the traditional point of view about this class of digraphs.
\end{abstract}

Keywords: Minimal strong digraphs, trees, characteristic polynomial, spanning tree. 


\section{Introduction}

A digraph is strongly connected or (simply) strong (SD) if every pair of vertices are joined by a path. An SD is minimal (MSD) if it loses the strong connection property when any of their arcs is suppressed. This class of digraphs has been considered under different points of view. See, for instance, $[4,6]$.

We are also interested in the following nonnegative inverse eigenvalue problem [8]: given $k_{1}, k_{2}, \ldots, k_{n}$ real numbers, find necessary and sufficient conditions for the existence of a nonnegative matrix $A$ of order $n$ with characteristic polynomial $x^{n}+k_{1} x^{n-1}+k_{2} x^{n-2}+\cdots+k_{n}$. The coefficients of the characteristic polynomial are closely related to the cycle structure of the weighted digraph with adjacency matrix $A$ (see, for instance, [5]). The class of strong digraphs can easily be reduced to the class of minimal strong digraphs, so we are interested in any theoretical or constructive characterization of these classes of digraphs.

In [6], a sequentially generative procedure for the constructive characterization of the classes of MSDs is given. In addition, algorithms to compute unlabeled MSDs and their isospectral classes are described. These algorithms have been implemented to calculate the said classes of digraphs up to order 15 classified by their order and size [10]. We are also interested in properties regarding the spectral structure of this class of digraphs, mainly about the coefficients of the characteristic polynomial.

MSDs can be seen as a generalization of trees, as we pass from simple graphs to directed graphs. Although the structure of MSDs is much richer than that of trees, many analogies remain between the properties of both families. Other properties, nevertheless, undergo radical changes when passing from trees to MSDs.

In this article, we focus on structural and spectral properties of MSDs. We carry out a comparative study of properties of MSDs versus trees. An extended version of this work can be found in [7].

\section{Minimal strong digraphs versus trees}

In this paper we use some standard basic concepts and results about graphs. A digraph $D$ is a couple $D=(V, A)$, where $V$ is a finite nonempty set and

\footnotetext{
1 Partially supported by MTM2015-65764-C3-1-P

2 Email: marijuan@mat.uva.es

3 Email: jglopez@etsisi.upm.es

4 Email: luispozo@etsisi.upm.es
} 
$A \subset V \times V-\{(v, v): v \in V\}$. Elements in $V$ and $A$ are called vertices and arcs respectively. If $u, v \in V$ we denote $(u, v)$ by $u v$. A path is a sequence of distinct vertices $v_{1} v_{2} \ldots v_{q}, q \geq 2$, such that $v_{i} v_{i+1}$ is an arc for $i=1,2, \ldots, q-1$. We denote a path from the vertex $u$ to the vertex $v$ by $u v$-path. A cycle of length $q$ or a $q$-cycle is a path $v_{1} v_{2} \ldots v_{q}$ closed by the arc $v_{q} v_{1}$. It is denoted by $C_{q}$. A double directed tree is the digraph obtained from a tree by replacing each edge $\{u, v\}$ with the two $\operatorname{arcs}(u, v)$ and $(v, u)$.

We now record a number of basic facts about the strong digraphs (see [6] and the references therein). In an SD of order $n \geq 2$, the in-degree and outdegree of the vertices are bigger than or equal to 1 . A vertex is linear if it has in- and out-degree equal to 1.

If $D$ is an MSD and there is a $u v$-path in $D$, then there cannot be an arc joining the vertex $u$ to the vertex $v$, that is $u v \notin A$. In general, an arc $u v$ in a digraph $D$ is transitive if there is another $u v$-path distinct from the arc $u v$. The semicycle consisting of a $u v$-path together with the arc $u v$ is a pseudocycle. So an MSD has no transitive arcs or pseudocycles; moreover, this condition characterizes the minimality of the strong connection. Consequently, if $D$ is an MSD then so is every strong subdigraph of $D$. Furthermore, every subdigraph that is an MSD is an induced subdigraph.

The contraction of a cycle of length $k$ in an SD consists of the reduction of the cycle to a unique vertex, so that $k-1$ of its vertices and its $k$ arcs are eliminated. The contraction obviously preserves the SD property.

Lemma 2.1 The contraction of a cycle in an MSD preserves the minimality, that is, it produces another $M S D$.

Lemma 2.2 The size of a minimal strong digraph $D$ of order $n \geq 2$ verifies $n \leq|A| \leq 2(n-1)$. The size of $D$ is $n$ if and only if $D$ is an n-cycle. The size of $D$ is $2(n-1)$ if and only if $D$ is a double directed tree.

Lemma 2.3 Every MSD of order $n \geq 2$ has at least two linear vertices.

Trees and MSDs are defined in a similar way. They are minimal connected graphs and minimal strong digraphs respectively, such that, in every case, the deletion of any edge and arc respectively implies connectivity loss. Despite the analogy in the definition, it is expected that the properties of these two kinds of graphs are very different because, while trees have no cycles, in every MSD, each arc belongs to a cycle.

However, surprisingly, there are many more analogies than differences between these two families of graphs. We explore the properties of both kind of graphs, so as to deeply understand the structure of MSDs, by using the very 
well-known family of trees.

Trees and MSDs have a linear number of edges and arcs respectively $m$, related to the number of vertices $n$. The order of a tree determines the number of edges, $m=n-1$, whereas this does not hold for MSDs. In this case, the number of arcs verifies $n \leq m \leq 2(n-1)$. On the other hand, considering an edge equivalent to two arcs, in both cases, the maximal number of arcs is $m=2(n-1)$.

There is an equivalent definition of trees: they are connected graphs with $n-1$ edges. This fact is related to the following property of MSDs: an strong digraph with $n$ arcs is an MSD (Lemma 2.2). Furthermore, the two families of graphs verify that they have at least two linear vertices, i.e. vertices with degree one and in- and out-degree one respectively (Lemma 2.3). Also, in both cases, there are configurations with a great number of linear vertices: tree stars have $n-1$ linear vertices and directed cycles have $n$; and with a vertex with high degree: both tree and MSD stars.

There are two other meaningful differences, besides the nonexistence and the existence of cycles. Given two vertices $u$ and $v$ in any tree, there is a unique $u v$-path. We denote this fact as path-tree property. Then, trees verify the path-tree property, while MSDs do not. If $u v$ is an arc in an MSD then this path is unique, but we cannot say the same in other cases. Nevertheless, there exists a subfamily of the MSD class where the property holds. It is defined below.

Definition 2.4 A directed cycle digraph is an SD in which every topological cycle is a directed cycle.

Here, a topological cycle means a cycle in the simple graph obtained by the substitution of every arc by an edge. Obviously, every directed cycle digraph is an MSD. However, we also state below that the class of MSDs satisfying the path-tree property is exactly the directed cycle digraph class.

Theorem 2.5 Let $D$ be an SD. Then, $D$ verifies the path-tree property if and only if $D$ is a directed cycle digraph.

The second meaningful difference between trees and MSDs is the complexity of the following algorithmic problem: Given a weighted connected graph and a weighted strong digraph, find the minimum spanning tree (MST) and the minimum spanning strong subdigraph (MSSS) respectively. While there are many polynomial algorithms to solve the MST problem [3], the MSSS problem belongs the the NP-hard class [1], even when all weights are one.

In the problem of covering the vertices of a graph, by paths or cycles, we 
come upon three new similar properties. We state below that an MSD has at most one covering by disjoint cycles. The corresponding property for trees says that every tree has at most one perfect matching. This property becomes the previous one if we consider edges equivalent to two arcs.

Theorem 2.6 If $D$ is an MSD then D has at most one covering by disjoint cycles.

The covering of a strong digraph $D$ with $\alpha$ cycles, not necessarily disjoint, where $\alpha$ is the stability number or the independent number of $D$, constitutes the Gallai conjecture. This was proved by Bessy and Thomassé [2] and the proof also applies to MSDs and trees if we consider that edges are equivalent to two arcs. There are examples of MSDs and trees where $\alpha$ cycles are needed in order to cover the corresponding digraph: MSD and tree stars.

The covering of a strong digraph $D$ with $\alpha-1$ disjoint paths, where $\alpha$ is, as above, the stability number or the independent number of $D$, constitutes the Las Vergnas conjecture. This was proved by Thomasse [9] and the proof also applies to MSDs and trees if we consider that edges are equivalent to two arcs. There are examples of MSDs and trees where $\alpha-1$ disjoint paths are needed to cover the corresponding digraph: MSD and tree stars.

Finally, we want to remark the following properties in which trees and MSDs behave in a similar way.

Let $D=(V, A)$ be an MSD with $n$ vertices and let $k_{m}(D)$ be the coefficient of $x^{n-m}$ in the monic characteristic polynomial of the adjacency matrix of $D$. Let $K_{m}(D)$ be the number of coverings of $m$ vertices (or arcs) in $D$ by disjoint cycles. Hence, $\left|k_{m}(D)\right| \leq K_{m}(D)$.

Theorem 2.7 Let $D$ be a double directed tree with $n$ vertices, $n \geq 2$. Then, $\left|k_{m}(D)\right|=K_{m}(D)$, and:

(i) For all $m$ odd such that $2 \leq m \leq n$, it is $K_{m}(D)=0$.

(ii) For all $m$ even such that $2 \leq m \leq n$, it is $K_{m}(D) \leq\left(\begin{array}{c}n-\frac{m}{2} \\ \frac{m}{2}\end{array}\right)$.

Conjecture 2.8 Let $D=(V, A)$ be an $M S D$ with $n$ vertices, $n \geq 2$, And $m$ an integer such that $2 \leq m \leq n$. Then, the following equality holds:

$$
K_{m}(D) \leq\left(\begin{array}{c}
n-\left\lceil\frac{m}{2}\right\rceil \\
\left\lfloor\frac{m}{2}\right\rfloor
\end{array}\right)
$$

The property that best explains the structure of an MSD is its factorization into two arborescences, the first with a unique source (a rooted tree), and the 
second with a unique sink in each connected component (a forest of reversed rooted trees).

Theorem 2.9 If $D$ is an $M S D$ then it factorises in a rooted spanning tree and a forest of reversed rooted trees.

\section{References}

[1] Bang-Jensen, J. and Gutin, G., Digraphs. Theory, Algorithms and Applications, Springer Monographs in Mathematics, Springer-Verlag, London, 2001.

[2] Bessy, S. and Thomassé, S., Spanning a strong digraph with alpha cycles: a conjecture of Gallai, Combinatorica 27(6) (2007) 659-667.

[3] Bondy, J.A. and Murty, U.S.R., Graph Theory, Springer, 2008.

[4] Brualdi, R. A. and Ryser, H. J., Combinatorial Matrix Theory, Cambridge University Press, New York, 1992.

[5] Cretkovic, D. M., Doob, M. and Sachs, H., Spectra of Graphs, Deutscher Verlag Wissenschaften, Berlin, 1982.

[6] García-López, J. and Marijuán, C., Minimal strong digraphs, Discrete Mathematics 312(4) (2012) 737-744.

[7] García-López, J., Marijuán, C. and Pozo-Coronado, L. M., Structural and spectral properties of minimal strong digraphs, preprint.

[8] Torre-Mayo, J., Abril-Raymundo, M. R., Alarcia-Estévez, E., Marijuán, C. and Pisonero, M., The nonnegative inverse eigenvalue problem from the coefficients of the characteristic polynomial, EBL digraphs, Linear Algebra Appl. 426 (2007) $729-773$.

[9] Thomassé, S., Covering a Strong Digraph by $\alpha-1$ Disjoint Paths: A Proof of Las Vergnas' Conjecture, Journal of Combinatorial Theory, Series B 83 (2001) 331-333.

[10] https://jglopez.etsisi.upm.es/MSC_Digraphs_Page/ 